\title{
A GENERAL DIFFERENTIAL INEQUALITY OF THE $k$ TH DERIVATIVE THAT LEADS TO NORMALITY
}

\author{
Qiaoyu Chen, Shahar Nevo and Xuecheng Pang \\ East China Normal University, Department of Mathematics \\ Shanghai 200241, P. R. China; goodluckqiaoyu@126.com \\ Bar-Ilan University, Department of Mathematics \\ Ramat-Gan 52900, Israel; nevosh@macs.biu.ac.il \\ East China Normal University, Department of Mathematics \\ Shanghai 200241, P. R. China; xcpang@math.ecnu.edu.cn
}

\begin{abstract}
Let $k \geq 0$ be an integer and $\alpha>1$. Let $\mathcal{F}$ be a family of functions meromorphic in a domain $D \subset \mathbf{C}$. If $\left\{\frac{\left|f^{(k)}\right|}{1+|f|^{\alpha}}: f \in \mathcal{F}\right\}$ is locally uniformly bounded away from zero, then $\mathcal{F}$ is normal.
\end{abstract}

\section{Introduction}

Recently, there has been renewed activity in the study of the connection between differential inequalities and normality. A natural point of departure for this subject is the well-known theorem due to Marty.

Marty's Theorem. [10, p. 75] A family $\mathcal{F}$ of functions meromorphic in a domain $D$ is normal if and only if $\left\{f^{\#}: f \in \mathcal{F}\right\}$ is locally uniformly bounded in $D$.

Following Marty's Theorem, Royden proved the following generalization.

Theorem R. [9] Let $\mathcal{F}$ be a family of functions meromorphic in a domain $D$ with the property that for each compact set $K \subset D$, there is a positive increasing function $h_{K}$ such that $\left|f^{\prime}(z)\right| \leq h_{K}(|f(z)|)$ for all $f \in \mathcal{F}$ and $z \in K$. Then $\mathcal{F}$ is normal in $D$.

This result has been significantly extended further in various directions; see [4], [11] and [13]. Li and Xie established a different kind of generalization of Marty's Theorem, which involves higher derivatives.

Theorem LX. [5] Let $\mathcal{F}$ be a family of functions meromorphic in a domain $D$ such that each $f \in \mathcal{F}$ has zeros only of multiplicities $\geq k, k \in \mathbf{N}$. Then $\mathcal{F}$ is normal in $D$ if and only if the family

$$
\left\{\frac{\left|f^{(k)}(z)\right|}{1+|f(z)|^{k+1}}: f \in \mathcal{F}\right\}
$$

is locally uniformly bounded in $D$.

doi:10.5186/aasfm.2013.3833

2010 Mathematics Subject Classification: Primary 30A10, 30D45.

Key words: Normal family, differential inequality.

Research of first and third author supported by the NNSF of China Approved No. 11071074.

Research of the second author is supported by the Israel Science Foundation, Grant No. 395/07. 
In [7], the second and the third authors gave a counterexample to the validity of Theorem LX, without the condition on the multiplicities of zeros for the case $k=2$.

Concerning differential inequalities with the reversed sign of the inequality, Grahl, and the second author proved the following result, which may be considered a counterpart to Marty's Theorem.

Theorem GN. [2] Let $\mathcal{F}$ be a family of functions meromorphic in $D$ and $C>0$. If $f^{\#}(z)>C$ for every $f \in \mathcal{F}$ and $z \in D$, then $\mathcal{F}$ is normal in $D$.

Steinmetz [12] gave a shorter proof of Theorem GN, using the Schwarzian derivative and some well-known facts on linear differential equations.

Then in [6], Liu together with the second and third authors generalized Theorem GN and proved the following result.

Theorem LNP. Let $1 \leq \alpha<\infty$ and $C>0$. Let $\mathcal{F}$ be the family of all meromorphic functions $f$ in $D$ such that

$$
\frac{\left|f^{\prime}(z)\right|}{1+|f(z)|^{\alpha}}>C
$$

for every $z \in D$.

Then the following hold:

(1) if $\alpha>1$, then $\mathcal{F}$ is normal in $D$;

(2) if $\alpha=1$, then $\mathcal{F}$ is quasi-normal in $D$ but not necessarily normal.

Observe that (2) of Theorem LNP is a differential inequality that distinguish between quasi-normality to normality.

In this paper, we continue to study differential inequalities with the reversed sign (" $\geq$ ") and prove the following general theorem.

Theorem 1. Let $D$ be a domain in C. Let $k \geq 0$ be an integer, $C>0, \alpha>1$ constants. Then the family $\mathcal{F}$ of all functions $f$ meromorphic in $D$ such that

$$
\frac{\left|f^{(k)}(z)\right|}{1+|f(z)|^{\alpha}}>C, \quad z \in D,
$$

is normal.

Let us set some notation. For $z_{0} \in \mathbf{C}$ and $r>0$ we put $\Delta\left(z_{0}, r\right)=\left\{z:\left|z-z_{0}\right|<\right.$ $r\}$ and $\bar{\Delta}\left(z_{0}, r\right)=\left\{z:\left|z-z_{0}\right| \leq r\right\}$. We write $f_{n}(z) \stackrel{\chi}{\Rightarrow} f(z)$ on $D$ to indicate that the sequence $\left\{f_{n}(z)\right\}$ converges to $f(z)$ in the spherical metric, uniformly on compact subsets of $D$, and $f_{n}(z) \Rightarrow f(z)$ on $D$ if the convergence is also in the Euclidean metric.

We need two lemmas for the proof.

\section{Auxiliary lemmas}

The first lemma we need is the lemma of Chen and $\mathrm{Gu}$ [1, Thm. 2], see also [8, Lemma 2]. Observe that this is an "if and only if" lemma.

Lemma 1. Let $\mathcal{F}$ be a family of functions meromorphic in a domain $D \subset \mathbf{C}$, all of whose zeros have multiplicity at least $m$, and all of whose poles have multiplicity at least $p$, and let $-p<\alpha<m$. Then $\mathcal{F}$ is not normal at some $z_{0} \in D$ if and only if there exist sequences $\left\{f_{n}\right\}_{n=1}^{\infty} \subset \mathcal{F},\left\{z_{n}\right\}_{n=1}^{\infty} \subset D,\left\{\rho_{n}\right\}_{n=1}^{\infty}$ satisfying $z_{n} \rightarrow z_{0}$, 
$\rho_{n} \rightarrow 0^{+}$and

$$
g_{n}(\xi):=\rho_{n}^{\alpha} f_{n}\left(z_{n}+\rho_{n} \xi\right) \stackrel{\chi}{\Rightarrow} g(\xi) \text { on } \mathbf{C},
$$

where $g$ is a nonconstant function meromorphic in $\mathbf{C}$.

The second lemma of which we shall make use is the general criterion of normality due to $\mathrm{Gu}$.

Lemma 2. [3] Let $k \geq 1$ be an integer. Then the family $\mathcal{F}$ of all functions meromorphic in a domain $\bar{D} \subset \mathbf{C}$ such that $f(z) \neq 0, f^{(k)}(z) \neq 1$ for every $z \in D$ is normal.

\section{Proof of Theorem 1}

The case $k=0$ is immediate, so we assume that $k \geq 1$. Let $z_{0} \in D$ and let $\left\{f_{n}\right\}_{n=1}^{\infty}$ be a sequence of functions of $\mathcal{F}$. We prove that $\left\{f_{n}\right\}_{n=1}^{\infty}$ is normal at $z_{0}$.

Separate into two cases.

Case (I). There is some $r>0$ and a subsequence of $\left\{f_{n}\right\}_{n=1}^{\infty}$, all of which are holomorphic in $\Delta\left(z_{0}, r\right)$.

Without loss of generality, we denote this subsequence also as $\left\{f_{n}\right\}_{n=1}^{\infty}$. Let us take $\beta>\frac{k}{\alpha-1}$.

If $\left\{f_{n}\right\}_{n=1}^{\infty}$ is not normal at $z_{0}$, then by Lemma 1 there is a subsequence of $\left\{f_{n}\right\}_{n=1}^{\infty}$ (that will also be denoted by $\left\{f_{n}\right\}_{n=1}^{\infty}$ ), and sequences $z_{n} \rightarrow z_{0}, \rho_{n} \rightarrow 0^{+}$such that

$$
\rho_{n}^{\beta} f_{n}\left(z_{n}+\rho_{n} \xi\right) \Rightarrow g(\xi) \text { on } \mathbf{C},
$$

where $g$ is a nonconstant entire function in $\mathbf{C}$.

Let $\xi_{0} \in \mathbf{C}$ be such that $g\left(\xi_{0}\right) \neq 0$. Differentiating (2) $k$ times at $\xi_{0}$ gives

$$
\rho_{n}^{\beta+k} f_{n}^{(k)}\left(z_{n}+\rho_{n} \xi_{0}\right) \underset{n \rightarrow \infty}{\longrightarrow} g^{(k)}\left(\xi_{0}\right) \text { on } \mathbf{C},
$$

By (2) and the choice of $\xi_{0}$ we have $f_{n}\left(z_{n}+\rho_{n} \xi_{0}\right) \underset{n \rightarrow \infty}{\longrightarrow} \infty$, and thus by (1) we have

$$
\left|f_{n}^{(k)}\left(z_{n}+\rho_{n} \xi_{0}\right)\right|>C\left|f_{n}\left(z_{n}+\rho_{n} \xi_{0}\right)\right|^{\alpha} .
$$

Thus $\rho_{n}^{\beta+k}\left|f_{n}^{(k)}\left(z_{n}+\rho_{n} \xi_{0}\right)\right|>C \rho_{n}^{\beta+k}\left|f_{n}\left(z_{n}+\rho_{n} \xi_{0}\right)\right|^{\alpha}=C\left(\rho_{n}^{\beta}\left|f_{n}\left(z_{n}+\rho_{n} \xi_{0}\right)\right|\right)^{\alpha} \rho_{n}^{\beta+k-\beta \alpha}$.

By the choice of $\beta$ and $\xi_{0}$ the last expression tends to $\infty$ as $n \rightarrow \infty$, and this is a contradiction to $(3)$, as $g^{(k)}\left(\xi_{0}\right)$ is finite.

Case (II). There are $N \in \mathbf{N}$ and $\left\{z_{n}\right\}_{n=N}^{\infty}$ such that $z_{n} \underset{n \rightarrow \infty}{\longrightarrow} z_{0}$ and $f_{n}\left(z_{n}\right)=\infty$. Without loss of generality $N=1$. Let $K_{n} \geq 1$ denote the multiplicity of the pole $z_{n}$ of $f_{n}$. We also assume that there is a sequence $\tilde{z}_{n} \underset{n \rightarrow \infty}{\longrightarrow} z_{0}$ such that $f_{n}\left(\tilde{z}_{n}\right)=0$. Indeed, by (1) we have $\left|f_{n}^{(k)}(z)\right|>C$ for every $n \geq 1$ and $z \in D$. If there was no such sequence $\tilde{z}_{n} \longrightarrow z_{0}$ as above, there would exist some $\rho>0$ and a subsequence of $\left\{f_{n}\right\}_{n=1}^{\infty}$ (that we also denote by $\left.\left\{f_{n}\right\}_{n=1}^{\infty}\right)$ such that $f_{n} \neq 0$ in $\Delta(0, \rho)$. Then by Lemma $2,\left\{f_{n}\right\}_{n=1}^{\infty}$ would be normal at $z_{0}$ and we are done.

Consider now the sequence $\left\{\frac{f_{n}^{(k)}}{f_{n}}\right\}_{n=1}^{\infty}$. If $\left|f_{n}(z)\right| \leq 1$, then $\left|\frac{f_{n}^{(k)}}{f_{n}}(z)\right| \geq\left|f_{n}^{(k)}(z)\right| \geq$ $\frac{\left|f_{n}^{(k)}(z)\right|}{1+\left|f_{n}(z)\right|^{\alpha}}>C$. If $\left|f_{n}(z)\right|>1$, then $\left|\frac{f_{n}^{(k)}}{f_{n}}(z)\right| \geq \frac{\left|f_{n}^{(k)}(z)\right|}{1+\left|f_{n}(z)\right|^{\alpha}}>C$. Hence $\left\{\frac{f_{n}^{(k)}}{f_{n}}\right\}_{n=1}^{\infty}$ is normal (in $D$ ) and so is $\left\{\frac{f_{n}}{f_{n}^{(k)}}\right\}_{n=1}^{\infty}$. Thus we can assume, after moving to a subsequence 
(that will also be denoted by $\left\{\frac{f_{n}}{f_{n}^{(k)}}\right\}_{n=1}^{\infty}$ ) that $\frac{f_{n}}{f_{n}^{(k)}} \underset{n \rightarrow \infty}{\Rightarrow} H$ in $D$. Since for each $n, \frac{f_{n}}{f_{n}^{(k)}}$ is holomorphic in $D$, and since $\frac{f_{n}}{f_{n}^{(k)}}\left(z_{n}\right)=\frac{f_{n}}{f_{n}^{(k)}}\left(\tilde{z}_{n}\right)=0, H$ is analytic in $D$. The point $\tilde{z}_{n}$ is a zero of $\frac{f_{n}}{f_{n}^{(k)}}$ of multiplicity at least 1 . The point $z_{n}$ is a zero of $\frac{f_{n}}{f_{n}^{(k)}}$ of multiplicity exactly $k$. Thus, if $H \not \equiv 0$, then by Rouche's Theorem $z_{0}$ is a zero of $H$ of multiplicity at least $k+1$. Thus, in both cases $H \not \equiv 0$ or $H \equiv 0$, we have

$$
\left(\frac{f_{n}}{f_{n}^{(k)}}\right)^{(k)}\left(z_{n}\right) \underset{n \rightarrow \infty}{\rightarrow} 0 .
$$

In some small neighborhood of $z_{n}$ (that depends on $n$ ), we have

$$
f_{n}(z)=\frac{A_{n}}{\left(z-z_{n}\right)^{K_{n}}}\left(1+h_{n}(z)\right)
$$

where $A_{n} \neq 0$ is a constant and $h_{n}$ is analytic, $h_{n}\left(z_{n}\right)=0$.

Differentiating (5) $k$ times gives

$$
f_{n}^{(k)}(z)=\frac{(-1)^{k} K_{n}\left(K_{n}+1\right) \cdots\left(K_{n}+k-1\right) A_{n}}{\left(z-z_{n}\right)^{K_{n}+k}}\left(1+h_{n}^{*}(z)\right),
$$

where $h_{n}^{*}$ has the same properties of $h_{n}$. Dividing (5) in (6) and differentiating $k$ times at $z_{n}$ gives

$$
\left(\frac{f_{n}}{f_{n}^{(k)}}\right)^{(k)}\left(z_{n}\right)=\frac{(-1)^{k} k !}{K_{n}\left(K_{n}+1\right) \cdots\left(K_{n}+k-1\right)} .
$$

Now, if $\left\{K_{n}\right\}_{n=1}^{\infty}$ is bounded, then the right hand side of (7) does not tend to 0 as $n \rightarrow \infty$, contradicting (4).

Otherwise, we can choose $n$ such that $K_{n}>\frac{k}{\alpha-1}$. We then have that both the nominator and the denominator of (1) are infinite at $z_{n}$ and by (6) we have

$$
\begin{aligned}
\frac{\left|f_{n}^{(k)}\left(z_{n}\right)\right|}{1+\left|f_{n}\left(z_{n}\right)\right|^{\alpha}} & =\lim _{z \rightarrow z_{n}} \frac{\frac{K_{n}\left(K_{n}+1\right) \cdots\left(K_{n}+k-1\right) \cdot\left|A_{n}\right|}{\left|z-z_{n}\right|^{K_{n}+k}}}{\frac{\left|A_{n}\right|^{\alpha}}{\left|z-z_{n}\right|^{K_{n} \alpha}}} \\
& =\lim _{z \rightarrow z_{n}}\left|A_{n}\right|^{1-\alpha} K_{n}\left(K_{n}+1\right) \cdots\left(K_{n}+k-1\right)\left|z-z_{n}\right|^{K_{n}(\alpha-1)-k} .
\end{aligned}
$$

By the choice of $K_{n}$ this limit is 0 . This is a contradiction to (1) and the proof of Theorem 1 is completed.

Acknowledgements. The authors thank the referee for his excellent report. The authors thank L. Zalcman for his useful comments.

\section{References}

[1] Chen, H. H., and Y.X. Gu: Improvement of Marty's criterion and its application. - Sci. China Ser. A 36:6, 1993, 674-681.

[2] Grahl, J., and S. Nevo: Spherical derivatives and normal families. - J. Anal. Math. 117, 2012, 119-128.

[3] Gu, Y.: A criterion for normality of families of meromorphic functions. - Sci. Sinica (special issue) $1,1979,267-274$.

[4] Hinkkanen, A.: Normal families and Ahlfors's five island theorem. - New Zealand J. Math. $22,1993,39-41$. 
[5] LI, S. Y., and H. XIE: On normal families of meromorphic functions. - Acta Math. Sin. 4, $1986,468-476$.

[6] Liu, X. J., S. Nevo, and X. C. PANG: Differential inequalities, normality and quasi-normality. - arXiv:1111.0844.

[7] Nevo, S., and X. C. PAng: A non explicit counterexample to a problem of quasi-normality. arXiv:1111.0841.

[8] Pang, X. C., and L. Zalcman: Normal families and shared values. - Bull. Lundon Math. Soc. $32,2000,325-331$.

[9] Royden, H. L.: A criterion for the normality of a family of meromorphic functions. - Ann. Acad. Sci. Fenn. Ser. A I Math. 10, 1985, 499-500.

[10] Schiff, J.: Normal families. - Springer, New York, 1993.

[11] Schwick, W.: On a normality criterion of H. L. Royden. - New Zealand J. Math. 23, 1994, 91-92.

[12] Steinmetz, N.: Normal families and linear differential equations. - J. Anal. Math. 117, 2012, $347-364$.

[13] Zalcman, L.: Normal families: new perspectives. - Bull. Amer. Math. Soc. (N.S.) 35, 1998, $215-230$.

Received 13 September 2012 • Revised received 22 December 2012 • Accepted 18 January 2013 